\title{
Vulnerability Identification and Resilience Enhancements of Urban Environments
}

\author{
K. Fischer ${ }^{\mathrm{a}}$, W. Riedel ${ }^{\mathrm{a}}$, I. Häring ${ }^{\mathrm{a}}$, A. Nieuwenhuijs ${ }^{\mathrm{b}}$, S. Crabbe ${ }^{\mathrm{c}}$, \\ S. Trojaborg ${ }^{\mathrm{d}}$, W. Hynes ${ }^{\mathrm{e}}$, I. Müllers ${ }^{\mathrm{f}}$ \\ ${ }^{a}$ Fraunhofer Institute for High-Speed Dynamics, Ernst-Mach-Institut \\ Efringen-Kirchen, Germany \\ ${ }^{\mathrm{b}}$ TNO, Defence, Safety and Security, JG The Hague, Netherlands \\ ${ }^{\mathrm{c}}$ Crabbe Consulting Ltd, Erfurt, Germany \\ ${ }^{\mathrm{d}}$ DISSING+WEITLING Architecture, Copenhagen, Denmark \\ ${ }^{\mathrm{e}}$ Downey Hynes Partnership, Dublin, Ireland \\ ${ }^{\mathrm{f}}$ Schüssler-Plan Ingenieurgesellschaft mbH, Düsseldorf, Germany
}

\begin{abstract}
Steadily increasing number of the world's population is living in urban centres. The issue of security and citizen safety in densely populated areas is a growing concern. Considering terrorism and large scale accident scenarios, natural disasters and crime, urban planning practice must be complemented with vulnerability identification and resilience enhancements methods.

The VITRUV project, funded by the European Commission under its FP7 Research \& Technical Development Programme, is carried out by a consortium of 12 industry partners, public end-users and research institutions drawn from 8 European countries. The aim is the development of software tools for the consideration of extraordinary threats. For the complex process of urban planning the tools supports the planning process through all three planning stages, from concept to plan and detail design, compatible with existing planning formats and software solutions. The qualitative or quantitative hazard and risk analysis of single buildings of infrastructure forms the basis. It consists of the analysis of events, scenarios, hazards, damage, frequency of events, exposure of personnel and risk including options for risk visualization and risk assessment for plan and detail level. Based on an all hazard risk approach, the tools will enable planners

- $\quad$ to include a security assessment and security knowledge in their planning process, in order to make well-considered systematic qualitative decisions (concept level),

- $\quad$ to analyse the susceptibility of urban spaces with respect to new threats (plan level), and

- $\quad$ to perform vulnerability analysis of urban spaces by computing the likely damage on humans, buildings and traffic infrastructure (detail level).
\end{abstract}




\section{Introduction}

Urban planners need support to make decisions concerning the aspects of security and safety planning. This decision support will be made with user friendly software tools in the EU-project VITRUV ${ }^{1}$. All three stages (see Fig. 1) will contribute to enable the development of more robust and resilient structures with respect to urban (re)planning, (re)design and/or (re)engineering. Planners who use VITRUV's tools will be able to deliver urban space less prone to and less affected by attacks and disasters, sustainably improving the security of citizens. Fig. 1 gives an overview of the operational stages of urban planning and the connection of each level to the software tools of the VITRUV-project.

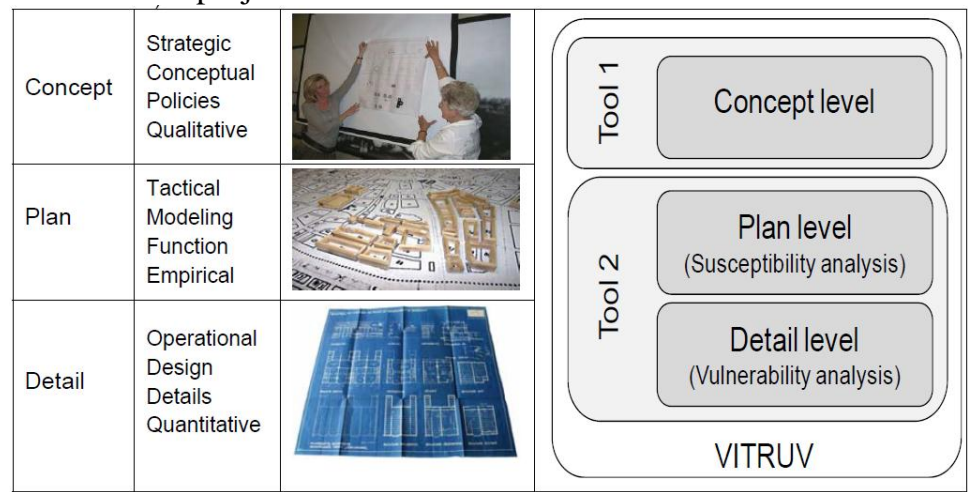

Fig. 1. Operational stages of urban planning (left) and tools overview of the VITRUV project (right).

\section{Overview of the tools}

This section gives a brief overview of the three stages of urban planning and the associated tools which will be developed during the VITRUV project.

\subsection{Concept level tool}

The aim of the concept level tool is to provide an easy-to-use, computer assisted support for urban planners in a systematic, qualitative way in order to make decisions with regard to the security of cities concerning specific problems or goals. Although the tools will specifically focus on the issue of security, it recognizes the fact that urban planners work in a holistic environment, i.e. that they have to consider a widely varied range of aspects and interests from a multitude of parties.

This is why the tool will have to place its security information and advice within this context: it will not only widen the knowledge of urban planners in security related issues, but will also place these in relationships with the other aspects of the urban

\footnotetext{
${ }^{1}$ Fraunhofer EMI, Vulnerability Identification Tools for Resilience Enhancements of Urban Environments, 2012, http://www.vitruv-project.eu
} 
planners' decision space, which will encompass among others, the economic, social, ethical, safety and mobility dimensions.

The concept tool is composed of two inter-related components: the Knowledge Base (Securipedia) and the Risk Assessment (Securban). These components work closely together in supporting the urban planner: the risk assessment components guides the urban planner via a short questionnaire as quickly as possible to potential security issues.

The tool will output the list of potential security issues in two ways:

- A prioritised (sorted) list of security issues that are deemed relevant for addressing in the project-issues with insufficient relevance will not be displayed.

- A complete (sequential) list of all possible security issues with an indication of its relevance to the project.

The prioritised list will allow the user to quickly get an overview of the issues to be dealt with. The complete list allows the user to quickly compare the results of two risk assessments. This can for example be achieved by presenting all possible security issues in a standard format, with the relevance expressed in a colour or bar indicator behind the issue. By manually overlaying two printed end results, the differences can quickly be found.

In the output of the Securban tool there are direct links to the Securipedia. Here they are explained and more background information can be found about the security issue and possible measures to mitigate the risk. In Securipedia, the security issue and its possible solutions are related to all the various aspects of an urban planning process, such as the impact on economy, mobility, social life, safety or ethics.

\subsection{Plan and detail level tool}

On the plan level of an urban planning process a susceptibility analysis will be carried out. This includes measures for frequency or probability of events based on specific building types. The susceptibility can be related to different quantities such as the frequency of (all/ selected) attack types for the considered building/ infrastructure types for example.

To get the empirical risk values for items of an urban area the frequency of events must be determined. Currently general crime and terrorist attacks are considered in this project. Further threats, like environmental disasters for example could be considered in future. In this paper examples concerning terrorist attacks will be presented.

To get empirical data of terrorist event the Fraunhofer EMI in-house database TED (Terror Event Database ${ }^{2}$ ) is used. The information from the TED are used in the project to determine the frequency of terrorist attacks, the tactic (e. g. car bomb), and the number of injuries and fatalities.

The empirical risk in dependency on the target (e.g. embassy or public place) is the result of this analysis. Hence on plan level the new tool can be used to analyse which parts of a city are mostly endangered.

\footnotetext{
${ }^{2}$ U. Siebold and I. Häring, Terror Event Database and Analysis Software, in Future Security, $4^{\text {th }}$ Security Research Conference, Karlsruhe, 2009
} 
The concept and plan level includes results for the guidance concerning risk assessment and an analysis concerning the susceptibility of targets in an urban area. In a next step the detail level is used to examine what are the effects of the threats that are identified during the plan level analysis. This examination will be carried out with a vulnerability analysis.

After the empirical risk analysis in the plan level the detail level tool includes algorithms for a quantitative risk assessment. The vulnerability is a measure for consequences risen from possible events. The vulnerability can be related to

- (local) average physical hazard in case of an attack,

- (local) average damage in case of an attack, and

- (local) average risk.

Within the approach the classic definition for risk computation is used. Therefore the quantitative risk is defined as a product of the frequency of an event and a measure for the consequences in the case the event happens.

The frequency is empirical determined during the plan level analysis. The consequences of considered threats can be calculated for persons and buildings. To calculate the structural response of buildings simplified engineering models are used. For this structural damage assessment more detailed information of the components are used, which are only available on detail level. Building costs and personal density are implemented to calculate lethality respectively structural damage and hence the derived risk for persons and buildings. The definition of the risk assessment on plan and detail level is precisely described and defined by $\operatorname{Voss}^{3}$.

These results are used for risk mapping. In combination with a three-dimensional visualization the user gets a quick overview for the considered urban area. In a next step security measures can be used to minimize the risk. The visualisation of plan and detail level occurs with a user-friendly Graphical User Interface (GUI).

\section{Conclusion}

In this paper the aims and the contents of the EU-project VITRUV are presented. During this project different risk assessment tools for urban planners are developed. The development of the software allows an identification, detailed analysis and resilience enhancement of "weak points" in urban areas. The tools can be used for planning and (re)design of urban areas to make them less prone for and less affected by terrorist attacks and natural disasters.

\section{Acknowledgements}

The research leading to these results has received funding from the European Commission's $7^{\text {th }}$ Framework Programme under grant agreement no. 261741. The contributions of all VITRUV consortium members are gratefully acknowledged.

\footnotetext{
${ }^{3}$ M. Voss, et.al.: ,Susceptibility and vulnerability of urban buildings and infrastructure against terroristic threats from qualitative and quantitative risk analyses", in European Safety and Reliability Association, ESREL Conference, Helsinki, 2012
} 